Pacific Journal of Mathematics

LIPCHITZ SPACES OF DISTRIBUTIONS ON THE SURFACE 


\title{
LIPSCHITZ SPACES OF DISTRIBUTIONS ON THE SURFACE OF UNIT SPHERE IN EUCLIDEAN $n$-SPACE
}

\author{
Harvey C. GReenwald
}

\begin{abstract}
In this paper Lipschitz spaces of distributions are defined and various inclusion relations are shown. Certain properties such as completeness, separability, and the density of the testing space for appropriate Lipschitz spaces are proved. The Littlewood-Paley function is defined and used to prove inclusion relationships between Lipschitz and Lebesgue spaces.
\end{abstract}

This paper is the second in a series of papers by the author of which [1] will be used extensively in this paper. As a result, a knowledge of [1] would be useful to the reader. In [1] the discussion was limited to Lipschitz spaces of functions. Here we extend the definition of a Lipschitz space to Distributions.

\section{Conventions and notation.}

$\mathbf{R}^{1}$ will denote the real numbers.

$\mathbf{R}^{n}=\left\{x=\left(x_{1}, \cdots, x_{n}\right): x_{i} \in \mathbf{R}^{1}, i=1, \cdots, n\right\}$.

$\Sigma_{n-1}=\left\{x \in \mathbf{R}^{n}:|x|=\left(x_{1}^{2}+\cdots+x_{n}^{2}\right)^{1 / 2}=1\right\}$. All functions are complex valued unless otherwise stated.

$C^{\infty}\left(\Sigma_{n-1}\right)$ is the set of indefinitely differentiable functions on $\Sigma_{n-1}$.

All statements about continuity, bounded, finiteness, etc., are made modulo sets of measure zero unless otherwise specified. By this we mean that a function that can be modified on a set of measure zero to have the property will be said to have the property.

If $f(x, r)$, where $x \in \Sigma_{n-1}$ and $0<r<1$, is differentiable with respect to $r$, we define $T f(x, r)=d / d r(r f)(x, r)$ and $T^{k} f(x, r)=T\left(T^{k-1} f\right)(x, r)$ where $k$ is an integer greater than 1 . We say $f(x)=0(g(x)), x \rightarrow a$, if $f(x) / g(x)$ is bounded as $x \rightarrow a$.

$$
\begin{aligned}
& f(x)=o(g(x)), x \rightarrow a, \text { if } f(x) / g(x) \rightarrow 0 \text { as } x \rightarrow a . \\
& f(x) \simeq g(x), x \rightarrow a, \text { if } f(x) / g(x) \rightarrow 1 \text { as } x \rightarrow a .
\end{aligned}
$$

For $\alpha$ real, $\bar{\alpha}$ will denote the smallest nonnegative integer larger than $\alpha$. If $f(x)$ is measurable on $\Sigma_{n-1}$, we define $\|f(x)\|_{p}=$

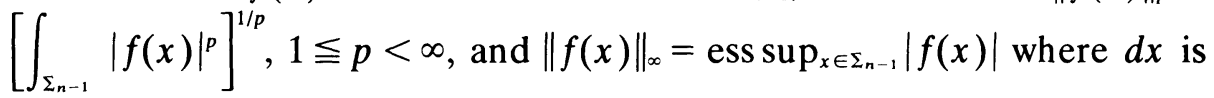
nonnormalized Lebesgue measure on $\Sigma_{n-1}$. If $f(x, r)$ is measurable in $x$ and $r$ where $x \in \Sigma_{n-1}$ and $0<r<1$, we define 


$$
\begin{aligned}
& \|f(r, x)\|_{p, d x}= \begin{cases}{\left[\int_{\Sigma_{n}, 1}|f(r, x)|^{p} d x\right]^{1 / p}} & \text { if } 1 \leqq p<\infty \\
\underset{x \in \Sigma_{n} 1}{\operatorname{ess~sup}}|f(r, x)| & \text { if } p=\infty\end{cases} \\
& \|f(r, x)\|_{p q}= \begin{cases}{\left[\int_{0}^{1}\|f(r, x)\|_{p, d x} d r / 1-r\right.} & \text { if } 1 \leqq q<\infty \\
\underset{0<r<1}{\operatorname{ess~sup}\|f(r, x)\|_{p, d x}} & \text { if } q=\infty .\end{cases}
\end{aligned}
$$

If $\alpha>0$ and $1 \leqq p, q \leqq \infty$, we say $f \in \Lambda(\alpha ; p, q)$ if

$$
\|f(x)\|_{\alpha ; p, q}=\|f(x)\|_{p}+\left\|(1-r)^{\alpha-\alpha} T^{\alpha} f(r, x)\right\|_{p q}
$$

is finite.

The Poisson kernel is the function $P(r x, y)=1 / c_{n}\left(1-r^{2}\right) /|r x-y|^{n}$ where $|x|=|y|=1, \quad 0 \leqq r<1, \quad$ and $c_{n}$ is a constant so that $\int_{\Sigma_{n-1}} P(r x, y) d y=1$ for each $x$. We shall also use $P(r, x \cdot y)=$ $1 / c_{n}\left(1-r^{2}\right) /\left[1-2 r x \cdot y+r^{2}\right]^{n / 2}$.

If $f(x) \in L_{p}\left(\Sigma_{n-1}\right), 1 \leqq p \leqq \infty$, then the Poisson integral of $f$ is defined as $f(r x)=\int_{\Sigma_{n-1}} f(y) P(r, x \cdot y) d y$.

$\left\{Y_{l}^{(k)}\right\}, l=1, \cdots, n(k)$, denotes an orthonormal basis for the spherical harmonics of degree $k$. $\quad Z_{y}^{(k)}$ denotes the zonal harmonic of degree $k$ with pole $y$.

If $F(x) \in L_{1}\left(\Sigma_{n-1}\right)$ and $G(s) \in L_{1}([-1,1], d \mu(s))$ where $d \mu(s)=$ $\left(1-s^{2}\right)^{(n-3) / 2}$, the spherical convolution of $F$ and $G$ is the function

$$
F * G(x)=\int_{\Sigma_{n-1}} F(y) G(x \cdot y) d y .
$$

Chapter I. Lipschitz Spaces, $\alpha$ Real.

In this chapter the notion of a Lipschitz space for $\alpha$ real is defined. For this a brief discussion of distributions is necessary.

Let the testing space $S=\left\{\phi: \phi \in C^{\infty}\left(\Sigma_{n-1}\right)\right\}$. Let $Y_{l}^{(k)}(x), \quad l=$ $1, \cdots, n(k)$, be an orthonormal basis for the spherical harmonics of degree k. $\phi \in C^{\infty}\left(\sum_{n-1}\right)$ if and only if $\phi=\sum_{k l} a_{k l} Y_{l}^{(k)}$ with $a_{k l}=0\left(k^{-s}\right)$ for all reals. For a proof of see Seeley [3]. $\phi$ can be considered to be in $C^{\infty}\left(\mathbf{R}^{n}-\{0\}\right)$ by noting that 


$$
\phi(x)=\sum_{k, l} a_{k l} Y_{l}^{(k)}(x)=\sum_{k, l} a_{k l} r^{-k} P_{l}^{(k)}(r x)
$$

where $P_{l}^{(k)}$ are harmonic polynomials of degree $k$.

Let $D^{\alpha} f=\partial^{|\alpha|} f / \partial x_{1}^{\alpha_{1}} \cdots \partial x_{n}^{\alpha_{n}}$ where $\alpha=\left(\alpha_{1}, \cdots, \alpha_{n}\right), \alpha_{i}$ nonnegative integers, and $|\alpha|=\alpha_{1}+\cdots+\alpha_{n}$. It is easy to see that $D^{\alpha} \phi=$ $\sum_{k, l} a_{k l} D^{\alpha} Y_{l}^{(k)}$ and the convergence is uniform. A topology can be defined on $S$ by letting

$$
N_{\epsilon, M}(0)=\left\{\phi \in C^{\infty}\left(\Sigma_{n-1}\right): \sum_{|\alpha| \leqq M}\left\|D^{\alpha} \phi\right\|_{\infty}<\epsilon\right\}
$$

be a neighborhood system at 0 . A standard argument shows that with respect to this norm, $S$ is complete.

Let the distributions $S^{\prime}$ be the set of continuous linear functionals on $S$. The action of $f \in S^{\prime}$ on $\phi \in S$ will be denoted by $f(\phi)$. If $\phi \in S$ and $\phi=\Sigma_{k, l} a_{k l} Y_{l}^{(k)}$, then the Poisson integral of $\phi$,

$$
\phi(r x)=\sum_{k, l} a_{k l} r^{k} Y_{l}^{(k)}(x)
$$

also is in $S$ for fixed $r<1$. It is easy to see that $\phi(r x) \rightarrow \phi(x)$ as $r \rightarrow 1^{-}$ in the topology of $S$.

If $f \in S^{\prime}$ and $P$ is the Poisson kernel, define $f * P$ by $(f * P)(\phi)=$ $f(P * \phi)$. We call this the Poisson integral of the distribution $f$. In view of the above, if $f, g \in S^{\prime}$ and if $f$ and $g$ have the same Poisson integral, they are equal as distributions.

If $f \in L_{p}\left(\Sigma_{n-1}\right), 1 \leqq p \leqq \infty, f$ defines a distribution by letting $f(\phi)=$ $\int_{\Sigma_{n-1}} f(x) \phi(x) d x$. If $f \in L_{p}\left(\Sigma_{n-1}\right), \quad$ define $J^{-\alpha} f$ by $J^{-\alpha} f(\phi)=$ $f\left(\sum_{k, l} a_{k l}(k+1)^{\alpha} Y_{l}^{(k)}\right)$ where $\alpha \geqq 0$ and $\phi=\Sigma_{k, l} a_{k l} Y_{l}^{(k)}$.

Clearly $\sum a_{k l}(k+1)^{\alpha} Y_{l}^{(k)} \in S$. An easy check shows that this does define a distribution. Moreover,

$$
J^{-\alpha} f(r x)=\left[\sum_{k=0}^{\infty}(k+1)^{\alpha} r^{k} Z_{e_{1}}^{(k)} * f\right](x)
$$

is the Poisson integral of $J^{-\alpha} f$.

In view of the above $J^{\alpha} f(r x)=\sum_{k, l} b_{k l} r^{k}(k+1)^{-\alpha} Y_{l}^{(k)}(x)$ for $\alpha$ real where $f \sim \sum_{k, l} b_{k l} Y_{l}^{(k)}$. This is easily seen to be harmonic on $\{Z \in$ $\left.\mathbf{R}^{n}:|Z|<1\right\}$.

The above definition of $J^{-\alpha} f$ agrees with the $J^{-\alpha} f$ defined in Proposition 5.10 of Greenwald [1] for $f \in \Lambda(\alpha+\beta ; p, q)$. Hence,

Proposition 1.1. $J^{\beta}$ maps $\Lambda(\alpha ; p, q)$ isomorphically onto $\Lambda(\alpha+\beta ; p, q)$ provided only $\alpha, \alpha+\beta>0,1 \leqq p, q \leqq \infty$. 
Definition. The Lebesgue space $L_{p, \alpha}\left(\Sigma_{n-1}\right)=\left\{f \in S^{\prime}: f=\right.$ $\left.J^{\alpha} \psi, \psi \in L_{p}\left(\Sigma_{n-1}\right)\right\}$ for $\alpha$ real, $1 \leqq p \leqq \infty$. Define $\|f\|_{p, \alpha}=\|\psi\|_{p}$. Thus $L_{p, \alpha}$ is a Banach space that is an isometric image of $L_{p}\left(\Sigma_{n-1}\right)$.

Definition. For $\alpha \leqq 0,1 \leqq p, q \leqq \infty$, let $\Lambda(\alpha ; p, q)$ be the set of distributions $f \in L_{p, \alpha-\frac{1}{2}}$ for which $\left\|(1-r)^{\bar{\alpha}-\alpha} T^{\bar{\alpha}} f(r x)\right\|_{p q}$ is finite. For $\alpha \leqq$ 0 , define $\|f\|_{\alpha ; p, q}=\|f\|_{p, \alpha-\frac{1}{2}}+\left\|(1-r)^{\bar{\alpha}-\alpha} T^{\bar{\alpha}} f(r x)\right\|_{p q}$.

Note. We are essentially defining $\Lambda(-\alpha ; p, q)$ to be $J^{-\alpha-\frac{1}{2}}\left[\Lambda\left(\frac{1}{2} ; p, q\right)\right]$. The choice of $\frac{1}{2}$ is arbitrary. Any $\beta>0$, would work as well.

REMARK. Let $\alpha$ be real and $1 \leqq p, q \leqq \infty$. Let $f \in L_{p, \alpha-\frac{1}{2}}$. Then if $k$ is any nonnegative integer greater than $\alpha$, the following norms are equivalent:

(i) $\left\|(1-r)^{k-\alpha} T^{k} f(r x)\right\|_{p q}+\|f\|_{p, \alpha-\frac{1}{2}}$

(ii) $\left\|(1-r)^{\bar{\alpha}-\alpha} T^{\bar{\alpha}} f(r x)\right\|_{p q}+\|f\|_{p, \alpha-\frac{1}{2}}$.

Proof. This is an immediate consequence of (2.3) of Greenwald [1].

Proposition 1.2. Let $\alpha$ be real and $1 \leqq p, q \leqq \infty$. Then the set of distributions $f \in L_{p, \alpha-\frac{1}{2}}$ for which $\left\|(1-r)^{\bar{\alpha}-\alpha} T^{\bar{\alpha}} f(r x)\right\|_{p q}<\infty$ normed with $(*)=\left\|(1-r)^{\bar{\alpha}-\alpha} T^{\alpha} f(r x)\right\|_{p q}+\|f\|_{p, \alpha-\frac{1}{2}}$ is topologically and algebraically equal to $\Lambda(\alpha ; p, q)$.

Proof. For $\alpha \leqq 0$ this is the definition. Hence it suffices to consider the case $\alpha>0$.

(a) Assume that $f \in L_{p, \alpha-\frac{1}{2}}$ and that $(*)<\infty$. We want to show that $f \in \Lambda(\alpha ; p, q)$. There is a $g \in L_{p}\left(\Sigma_{n-1}\right)$ such that

$$
f(r x)=\left[g * G^{\alpha-\frac{1}{2}}(r, \cdot)\right](x) .
$$

Hence $\|f(r x)\|_{p, d x} \leqq M_{\alpha}\|g\|_{p}=M_{\alpha}\|f\|_{p, \alpha-\frac{1}{2}}$ if $r \leqq \frac{1}{2}$.

By Proposition 5.1 of Greenwald [1], $f(r x)$ is the Poisson integral of a function $h \in L_{p}\left(\Sigma_{n-1}\right)$ and $\|h\|_{p} \leqq M_{\alpha}(*)$. Now $h$ and $f$ have the same Poisson integral and thus are equal as distributions. Hence $f$ is a function and is in $L_{p}\left(\Sigma_{n-1}\right)$. Therefore $f \in \Lambda(\alpha ; p, q)$ and $\|f\|_{\alpha ; p, q} \leqq M(*)$.

(b) Let $f \in \Lambda(\alpha ; p, q)$. The proof of this part is essentially the same as that for Lemma 8 of Taibleson [18; p. 438].

The proofs of the following Propositions 1.3, 1.4, 1.5, and 1.6 are analogous to the proofs of Theorems $6,7,8$, and 9 of Taibleson [7; p. 437-443]. The appearence of the $(n-1)$ in Proposition 1.6 comes from the estimate $\|P((1+r) / 2 x)\|_{p} \leqq A(1-r)^{(1-n) / t^{\prime}}$ where $1 / t+2 / t^{\prime}=1$. See Greenwald $[1 ;(1.8)]$. 
Proposition 1.3. Let $\alpha, \beta$ be real and $1 \leqq p, q \leqq \infty$. Then $J^{\beta}$ maps $\Lambda(\alpha ; p, q)$ isomorphically onto $\Lambda(\alpha+\beta ; p, q)$.

Proposition 1.4. Let $f \in S^{\prime}$ and $f(r x)$ be its Poisson integral. Then for each integer $k \geqq \bar{\alpha}$ and real number $\beta<\alpha$, the norm

$$
\left\|(1-r)^{k-\alpha} T^{k} f(r x)\right\|_{p q}+\|f\|_{p, \beta}
$$

is equivalent to $\|f\|_{\alpha, p, q}$ where $1 \leqq p, q \leqq \infty$.

Proposition 1.5. Suppose $f \in \Lambda\left(\alpha_{i} ; p_{i}, q_{i}\right)$ where $i=0,1$ and $1 \leqq p_{i}$, $q_{1} \leqq \infty$. Let $0 \leqq t \leqq 1$ and define $\alpha=(1-t) \alpha_{0}+t \alpha_{1}, \quad 1 / p=$ $(1-t) / p_{0}+t / p_{1}$, and $1 / q=(1-t) q_{0}+t / q_{1}$. Then $f \in \Lambda(\alpha ; p, q)$ and

$$
\|f\|_{\alpha, p, q} \leqq M_{\alpha_{1}, \alpha_{1}}\left(\|f\|_{\alpha_{1}, p_{1}, q_{0}}\right)^{1-t}\left(\|f\|_{\alpha_{1} ; p_{1}, q_{1}}\right)^{t}
$$

Also, (i) $\|f\|_{p, \beta} \leqq\|f\|_{p_{0}, \beta}^{1-t}\|f\|_{p_{1}, \beta}$ for $\beta<\alpha_{0}, \alpha_{1}$.

(ii) $\left\|(1-r)^{k-\alpha} T^{k} f(r x)\right\|_{p q}$ $\leqq\left(\left\|(1-r)^{k-\alpha_{0}} T^{k} f(r x)\right\|_{\left.p_{1} q_{1}\right)}\right)^{1-t}\left(\left\|(1-r)^{k-\alpha_{1}} T^{k} f(r x)\right\|_{p_{1} q_{1}}\right)^{t}$ for $k<\alpha_{0}, \alpha_{1}$.

Proposition 1.6. Let $1 \leqq p_{1} \leqq q_{1} \leqq \infty$. Then $\quad \Lambda\left(\alpha_{1} ; p_{1}, q_{1}\right) \subset$ $\Lambda\left(\alpha_{2} ; p_{2} q_{2}\right)$ if $\alpha_{1}-(n-1) / p_{1}>\alpha_{2}-(n-1) / p_{2}$ or if $\alpha_{1}-(n-1) / p_{1}=$ $\alpha_{2}-(n-1) / p_{2}$ and $1 \leqq q_{1} \leqq q_{2} \leqq \infty$. Moreover, the inclusion map is continuous.

Proposition 1.7. (i) $\Lambda(\alpha ; p, q)$ is complete if $1 \leqq p, q<\infty$. (ii) $S$ is dense in $\Lambda(\alpha ; p, q)$ if $1 \leqq p \leqq \infty, 1 \leqq q<\infty$. We shall need the following lemma.

Lemma 1.1. If $f \in \Lambda(\alpha ; \infty, q), 0<\alpha<1$, then $f$ is uniformly continuous.

Proof. It suffices to show that $\|f(r x)-f(x)\|_{x, d x} \rightarrow 0$ as $r \rightarrow 1^{-}$. By Proposition 1.6, $f \in \Lambda(\alpha ; \infty, \infty) . \quad f(r x)-f(x)=\int_{r}^{1} f_{\rho}(\rho x) d \rho$ for almost every $x \in \Sigma_{n-1}$. Hence $\|f(r x)-f(x)\|_{x, d x} \leqq 2 \int_{r}^{1}\left\|\rho f_{\rho}(\rho x)\right\|_{x, d x} d \rho$ if $r \geqq$ $\frac{1}{2}$. Thus

$$
(1-r)^{-\alpha}\|f(r x)-f(x)\|_{x, d x} \leqq 2(1-r)^{-\alpha} \int_{r}^{1} A(1-\rho)^{\alpha-1} d \rho \leqq M_{\alpha} .
$$

Hence $\|f(r x)-f(x)\|_{x, d x} \leqq M_{\alpha}(1-r)^{\alpha}$ and thus tends to zero as $r \rightarrow 1^{-}$. 
Proof of Proposition 1.7. (i) The proof is similar to the proof of the corresponding part of Theorem 11 of Taibleson [7; p. 444]. (ii) It is easy to see that $J^{\alpha} S=S$. Hence, it suffices to consider the case $0<\alpha<1$. We claim that $S \subset \Lambda(\alpha ; p, q)$. Let $\phi \in S$ with $\phi=\Sigma_{k, l} a_{k l} Y_{l}^{(k)}$. Then $\phi(r x)=$ $\sum_{k, l} a_{k l} r^{k} Y_{l}^{(k)}(x)$ and $T \phi(r x)=\sum_{k, l} a_{k l}(k+1) r^{k} Y_{l}^{(k)}(x)$.

Since $\quad a_{k l}=O\left(k^{-s}\right) \quad$ for every $\quad s>0, \quad|T \phi(r x)| \leqq$ $\sum_{k, l}\left|a_{k l}(k+1) Y_{l}^{(k)}\right| r^{k} \leqq M<\infty$. So, $\quad \phi \in \Lambda(\alpha ; p, q)$. Let $f \in$ $\Lambda(\alpha ; p, q)$. If $1 \leqq p<\infty, f(r x) \rightarrow f(x)$ in $L_{p}\left(\Sigma_{n-1}\right)$ as $r \rightarrow 1^{-}$.

If $p=\infty, f$ is continuous by Lemma 1.1 and so $f(r x) \rightarrow f(x)$ in $L_{x}\left(\Sigma_{n-1}\right)$ as $r \rightarrow 1^{-}$. We claim that for each $r,(1-r)^{1-\alpha} \| T f(r s x)-$ $T f(r x) \|_{p, d x} \rightarrow 0$ as $s \rightarrow 1^{-}$.

Let $g(x)=T f(r x)$. Then $g(s x)=T f(r s x)$. By the above, $g(s x) \rightarrow g(x)$ in $L_{p}\left(\Sigma_{n-1}\right)$ as $s \rightarrow 1^{-}$. Also, $\|T f(r s x)\|_{p, d x} \leqq\|T f(r x)\|_{p, d x}$.

If $q<\infty$, by applying the Dominated Convergence Theorem we have that $\|(1-r)^{1-\alpha}\left[T f(r s x)-T f(r x] \|_{p q} \rightarrow 0\right.$ as $s \rightarrow 1^{-}$.

Thus $f(s x) \rightarrow f(x)$ in $\Lambda(\alpha ; p, q)$ if $q<\infty$. For fixed $s<1, f(s x)$ is clearly in $S$. This finishes the proof.

Remarks. Let $A=\left\{\phi \in S: a_{k l}\right.$ are rational $\}$. It is clear that $A$ is dense in $\Lambda(\alpha ; p, q)$ if $q<\infty$. Hence $\Lambda(\alpha ; p, q)$ is separable if $q<\infty$.

Let $B=\left\{\alpha \in S: \Sigma_{k, l} a \Sigma_{k, l} a_{k l} Y_{l}^{(k)}\right.$ consists only of a finite number of terms $\}$. It is clear that $B$ is dense $\Lambda(\alpha ; p, q)$ if $q<\infty$.

Lastly, before finishing this chapter we would like to relate the Lipschitz spaces defined here to those defined by Ragozin [2]. For this we will need the notion of a derivative in the distribution sense.

Definition. If $f$ is differentiable on $\Sigma_{n-1}$ and $D$ is a skewsymmetric $n \times n$ matrix then we define $D f(x)=\left.(d / d t) f[(\exp t D)(x)]\right|_{t=0}$.

See Rogazin [2] for a more complete discussion of this topic.

Let $f, \phi \in S$. Then an easy argument shows that $\int_{\Sigma_{n-1}} D f(x) \phi(x) d x=\int_{\Sigma_{n-1}} f(x)(-D) \phi(x) d x$. This leads to the following definition.

Definition. Let $f \in S^{\prime}$. We define $D f$ to be the distribution defined by $D f(\phi)=f(-D \phi)$ for $\phi \in S$.

If $i<j$, let $D_{i j}$ be the $n \times n$ matrix with a 1 in the $(i, j)$ place, a $(-1)$ in the $(j, i)$ place, and zeros everywhere else. See Ragozin [2].

Definition. We define $\left(D_{i j}\right)^{k}$ to be the result of applying some $k$-fold product of $D_{i j}$ 's to $f$ in the distribution sense where $f \in S^{\prime}$. This is clearly ambiguous but we shall be summing over all possible $k$-fold products. Hence, no problem will arise. 
Proposition 1.8. Let $\alpha>0$ and $1 \leqq p, q \leqq \infty$. Then the norm $\|f(x)\|_{p}+\Sigma\left\|\left(D_{l j}\right)^{k} f\right\|_{\alpha, p, q}$ is equivalent to $\|f\|_{\alpha+k, p, q}$ where the sum is over all $k$-fold products of the $D_{i j}$ 's.

Proof. The proof is essentially the same as that used by Taibleson for the analogous result in $\mathbf{R}^{n}$. See Taibleson [7; Theorem 10, $\mathrm{p}$. 444]. The proof uses (1.3) and Proposition 5.1 of Greenwald [1].

Chapter II. The Relation of Lipschitz Spaces to Lebesgue Spaces.

In this chapter certain inclusion relations between Lipschitz Spaces and Lebesgue Spaces are proved. The chief tool is an analogue of the Littlewood-Paley function. The proofs are, for the most part, direct analogues of those used by Stein [4], [5] for the Littlewood-Paley function in a compact Lie group. The reader is referred to Zygmund [8; Chapter XIV] for the one dimensional version of the Littlewood-Paley function.

DEFINITION. Let $f^{*}(x)=\sup _{0<t \leqq \pi} t^{-n+1} \int_{d(x, y) \leqq t}|f(y)| d y \quad$ if $f \in L_{1}\left(\Sigma_{n-1}\right)$.

REMARK. If $f \in L_{p}\left(\Sigma_{n-1}\right) ; 1<p \leqq \infty$, then $f^{*} \in L_{p}\left(\Sigma_{n-1}\right)$ and $\left\|f^{*}\right\|_{p} \leqq$ $A_{p, n}\|f\|_{p}$.

(2.1) Let $f \in L_{1}\left(\Sigma_{n-1}\right)$. Then $\sup _{0 \leqq r<1}|f(r x)| \leqq A_{n} f^{*}(x)$ for all $x \in \Sigma_{n-1}$.

Proof. We may assume that $f(x) \geqq 0$. Let $x \in \Sigma_{n-1}$ and let $g(\theta)=$ $\int_{x \cdot y=\cos \theta} f(y) d y$ and $G(t)=\int_{0}^{t} \sin ^{n-2} \theta g(\theta) d \theta$.

The proof now proceeds in a manner similar to the proof of Theorem 3.4 of Stein and Weiss [6; p. 101].

$$
\text { If } f \in L_{1}\left(\Sigma_{n-1}\right) \text {, then } \sup _{0<r<1} r \ln (1 / r)\left|(r f)_{r}(r x)\right| \leqq A_{n} f^{*}(x) .
$$

Proof.

$$
\begin{aligned}
r \ln (1 / r)\left|(r f)_{r}(r x)\right| & \leqq r \ln (1 / r) \int_{\Sigma_{n-1}}|f(y)|\left|(r P)_{r}(r x \cdot y)\right| d y \\
& \leqq B_{n} \int_{\Sigma_{n-1}}|f(y)||P(r x \cdot y)| d y \leqq A_{n} f^{*}(x)
\end{aligned}
$$

by (2.1) above. 
(2.3) If $f \in L_{1}\left(\Sigma_{n-1}\right)$, then $|\nabla f(r x)| \leqq A_{n} f^{*}(x)$ if $r \leqq \frac{1}{2}$ where $\nabla$ is the gradient in $\mathbf{R}^{n}$.

Proof. The proof is similar to proof of (2.2) above.

(2.4) Let $f \in L_{p}\left(\Sigma_{n-1}\right)$ and $F(r x)=[f(r x)]^{p}$ where $1<p<$ $\infty$. Then $\left|F_{r}(r x)\right| \leqq A_{p n}(1-r)^{-1}\left(f^{*}(x)\right)^{p}$.

Proof. $\quad F_{r}(r x)=p[f(r x)]^{p-1} f_{r}(r x)$. As in (2.2), $\quad\left|f_{r}(r x)\right| \leqq$ $A_{n}(1-r)^{-1} f^{*}(x)$. The result now easily follows.

Definition. Let $f \in L_{1}\left(\Sigma_{n-1}\right)$. Define

$$
g(f)(x)=\left[\int_{0}^{1} r \ln (1 / r)|\nabla f(r x)|^{2} d r\right]^{1 / 2} .
$$

Remark. The map $f \rightarrow g(f)$ is sublinear. This will enable us to apply the Marcinkiewicz Interpolation Theorem later in the chapter.

Proposition 2.1. Let $f \in L_{p}\left(\Sigma_{n-1}\right), 1<p<2$. Then $g(f) \in L_{p}\left(\Sigma_{n-1}\right)$ and $\|g(f)(x)\|_{p} \leqq A_{p, n}\|f\|_{p}$.

Proof. It suffices to prove this for $f$ strictly positive. In view of (2.3) we need only consider $\left[\int_{1 / 2}^{1} r \ln (1 / r)|\nabla f(r x)|^{2} d r\right]^{1 / 2}$.

Let $F(r x)=[f(r x)]^{p}$. An easy calculation shows that $|\nabla f(r x)|^{2}=$ $p^{-1}(p-1)^{-1}[f(r x)]^{2-p} \Delta\left[(f(r x))^{p}\right]$. So

$$
\begin{aligned}
\int_{1 / 2}^{1} r \ln (1 / r)|\nabla f(r x)|^{2} d r & =p^{-1}(p-1)^{-1} \int_{1 / 2}^{1} r \ln (1 / r)[f(r x)]^{-p} \Delta F(r x) d r \\
& \leqq\left[f^{*}(x)\right]^{2-p} A_{p, n} \int_{1 / 2}^{1} r \ln (1 / r) \Delta F(r x) d r .
\end{aligned}
$$

Let $I(x)=\int_{1 / 2}^{1} r \ln (1 / r) \Delta F(r x) d r$

$$
\begin{aligned}
\int_{\Sigma_{n-1}} I(x) d x & =\int_{1 / 2}^{1} r \ln (1 / r) \int_{\Sigma_{n-1}} \Delta F(r x) d x d r \\
& =\int_{1 / 2}^{1} r \ln (1 / r) \int_{\Sigma_{n-1}} r^{-n+1} \frac{\partial}{\partial r}\left(r^{n-1} \frac{\partial}{\partial r} F(r x)\right) d x d r \\
& =\int_{\Sigma_{n-1}} \int_{1 / 2}^{1} r \ln (1 / r) r^{-n+1} \frac{\partial}{\partial r}\left(r^{n-1} \frac{\partial}{\partial r} F(r x)\right) d r d x
\end{aligned}
$$


Now

$$
\begin{aligned}
& \int_{1 / 2}^{1} r \ln (1 / r) r^{-n+1} \frac{\partial}{\partial r}\left(r^{n-1} \frac{\partial}{\partial r} F(r x)\right) d r \\
& =\int_{1 / 2}^{1} r \ln (1 / r) F_{r r}(r x) d r+(n-1) \int_{1 / 2}^{1} \ln (1 / r) F_{r}(r x) d r .
\end{aligned}
$$

The second integral is bounded by $B_{p, n} f^{*}(x)^{p}$ by (2.4) since $\ln (1 / r) \leqq$ $A(1-r)$ if $r \geqq 1 / 2$. Upon integrating the first by parts, one gets

$$
\left|\int_{1 / 2}^{1} r \ln (1 / r) r^{-n+1} \frac{\partial}{\partial r}\left(r^{n-1} \frac{\partial}{\partial r} F(r x)\right) d r\right| \leqq C_{p, n}\left[f^{*}(x)\right]^{p} .
$$

Thus

$$
\int_{\Sigma_{n-1}} I(x) d x \leqq C_{p, n} \int_{\Sigma_{n-1}}\left(f^{*}(x)\right)^{p} d x \leqq C_{p, n}^{\prime}\|f\|_{p}^{p}
$$

Hence

$$
\begin{aligned}
\left\|\left[\int_{1 / 2}^{1} r \ln (1 / r)|\nabla f(r x)|^{2} d r\right]^{1 / 2}\right\|_{p} & \leqq A_{p, n}\left[\int_{\Sigma_{n-1}}\left[f^{*}(x)\right]^{1 / 2(2-p) p}[I(x)]^{p / 2} d x\right]^{1 / p} \\
& \leqq A_{p, n}\left\|f^{*}(x)\right\|_{p}^{1 / 2(2-p) p}\|f\|_{p}^{p^{2 / 2}}
\end{aligned}
$$

by Hölder's inequality.

The above is bounded by $A_{p, n}^{\prime \prime}\|f\|_{p}$. The result for an arbitrary $f$ now follows from an easy limiting argument.

Proposition 2.2. Let $f \in L_{p}\left(\Sigma_{n-1}\right), \quad 2 \leqq p<\infty$. Then $g(f) \in$ $L_{p}\left(\Sigma_{n-1}\right)$ and $\|g(f)\|_{p} \leqq A_{p, n}\|f\|_{p}$.

Proof. By the Marcinkiewicz Interpolation Theorem it suffices to prove this for $p \geqq 4$. Choose $q$ such that $q^{-1}+(p / 2)^{-1}=1$. Then $1<q \leqq 2 .\|g(f)\|_{p}^{2}=\sup \int_{\Sigma_{n-1}} g^{2}(f)(x) h(x) d x$ where the sup is over all $h \geqq 0$ such that $h \in L_{q}\left(\Sigma_{n-1}\right)$ and $\|h\|_{q}=1$.

$$
\int_{\Sigma_{n-1}} g^{2}(f)(x) h(x) d x=\int_{\Sigma_{n-1}}\left[\int_{0}^{1} r \ln (1 / r)|\nabla f(r x)|^{2} d r\right] h(x) d x .
$$

Let $w(r x)=|\nabla f(r x)|^{2}$. Since each partial derivative is harmonic, $w$ is subharmonic. Thus $w(r \rho x) \leqq \int_{\Sigma_{n-1}} w(\rho y) P(r x \cdot y) d y$ since the right 
side is harmonic in $r x$ and converges to the continuous function $w(\rho x)$ as $r \rightarrow 1^{-}$. Hence $w\left(r^{2} x\right) \leqq \int_{\Sigma_{n-1}} w(r y) P(r x \cdot y) d y$. Hence

$$
\int_{\Sigma_{n-1}} g^{2}(f)(x) h(x) d y \leqq 4 \int_{\Sigma_{n-1}}\left[\int_{0}^{1} r^{3} \ln (1 r)|\nabla f(r x)|^{2} h(r x) d r\right] d x .
$$

Now $|\nabla f(r x)|^{2}=\frac{1}{2} \Delta f^{2}(r x)$ and

$$
\Delta\left[f^{2}(r x) h(r x)\right]=f^{2}(r x) \Delta h(r x)+h(r x) \Delta f^{2}(r x)+\sum_{i=1}^{n} \frac{\partial}{\partial x_{i}} f^{2}(r x) \frac{\partial}{\partial x_{i}} h(r x)
$$

where $x_{1}, \cdots, x_{n}$ are the Cartesian Coordinates. Since $h(r x)$ is harmonic, $\Delta h=0$. Thus,

$$
\begin{aligned}
4 \int_{\Sigma_{n-1}} & {\left[\int_{0}^{1} r^{3} \ln (1 / r)|\nabla f(r x)|^{2} h(r x) d r\right] d x } \\
\leqq & 2\left|\int_{\Sigma_{n-1}}\left[\int_{0}^{1} r^{3} \ln (1 / r) \Delta\left[f^{2}(r x) h(r x)\right] d r\right] d x\right| \\
& +4 n \int_{\Sigma_{n-1}}\left[\int_{0}^{1} r^{3} \ln (1 / r)|f(r x)||\nabla f(r x)||\nabla h(r x)| d r\right] d x .
\end{aligned}
$$

The second integral is bounded by $A_{n} \int_{\Sigma_{n-1}} f^{*}(x) g(f)(x) g(h)(x) d x$ by Schwartz's Inequality since $|f(r x)| \leqq A_{n}^{\prime} f^{*}(x)$. By Hölder's Inequality, this integral is bounded by $A_{p, n}\left\|f^{*}\right\|_{p}\|g(f)\|_{p}\|g(h)\|_{q} \leqq$ $A_{p, n}^{\prime}\|f\|_{p}\|g(f)\|_{p}$ since $\|g(h)\|_{q} \leqq B_{n, q}\|h\|_{q}=B_{n, q}$ for $1<q \leqq 2$. The first integral equals

$$
I=\mid \int_{\Sigma_{n-1}}\left[\int_{0}^{1} r^{3} \ln (1 / r) r^{-n+1} \frac{\partial}{\partial r}\left(r^{n-1} \frac{\partial}{\partial r}\left[f^{2}(r x) h(r x)\right] d r\right] d x \mid\right.
$$

since spherical part of the Laplacian vanishes.

$$
\begin{aligned}
& \int_{0}^{1} r^{3} \ln (1 / r) r^{-n+1} \frac{\partial}{\partial r}\left[r^{n-1} \frac{\partial}{\partial r} f^{2}(r x) h(r x)\right] d r \\
& =\int_{0}^{1} r^{3} \ln (1 / r) \frac{\partial^{2}}{\partial r^{2}}\left(f^{2}(r x) h(r x)\right) d r \\
& \quad+(n-1) \int_{0}^{1} r^{2} \ln (1 / r) \frac{\partial}{\partial r}\left(f^{2}(r x) h(r x)\right) d r .
\end{aligned}
$$

In the second integral use $\left|r \ln (1 / r) f_{r}(r x)\right| \leqq B_{n} f^{*}(x)$, $\left|r \ln (1 / r) h_{r}(r x)\right| \leqq B_{n} h^{*}(x),|f(r x)| \leqq B_{n} f^{*}(x)$, and $|h(r x)| \leqq B_{n} h^{*}(x)$. 
Hence, the second integral is bounded by $B_{n}^{\prime} f^{*}(x)^{2} h^{*}(x)$. The first integral equals

$$
\begin{aligned}
\left.r^{3} \ln (1 / r) \frac{\partial}{\partial r}\left(f^{2}(r x) h(r x)\right)\right|_{0} ^{1}-\int_{0}^{1}\left[3 r^{2} \ln (1 / r)-r^{2}\right] \frac{\partial}{\partial r}\left(f^{2}(r x) h(r x)\right) d r \\
=\left.r^{3} \ln (1 / r) \frac{\partial}{\partial r}\left[f^{2}(r x) h(r x)\right]\right|_{0} ^{1} \\
-\left.\left[3 r^{2} \ln (1 / r)-r^{2}\right]\left[f^{2}(r x) h(r x)\right]\right|_{0} ^{1} \\
+\int_{0}^{1}[6 r \ln (1 / r)-5 r]\left[f^{2}(r x) h(r x)\right] d r .
\end{aligned}
$$

Using the above estimates one gets that the absolute value is bounded by $B_{n}^{\prime \prime} f^{*}(x)^{2} h^{*}(x)$. So

$$
I \leqq B_{n}^{\prime \prime \prime} \int_{\Sigma_{n-1}} f^{*}(x)^{2} h(x) d x \leqq B_{n, p}\left\|f^{*}\right\|_{p}^{2}\left\|h^{*}\right\|_{q} \leqq C_{n, p}\|f\|_{p}^{2}
$$

Thus $\quad\|g(f)\|_{p}^{2} \leqq A_{p, n}^{\prime}\|f\|_{p}\|g(f)\|_{p}+C_{n, p}\|f\|_{p}^{2}$. Hence $\quad\|g(f)\|_{p} \leqq$ $C_{n, p}^{\prime}\|f\|_{p}$

DEFINITION.

$$
g_{p}(f)(x)=\left[\int_{0}^{1}\left(r \ln (1 / r)\left|(r f)_{r}(r x)\right|\right)^{p} d r /(r \ln (1 / r))\right]^{1 / p}
$$

for $f(r x)$ harmonic on $B_{n}$ if $1 \leqq p<\infty$.

$$
g_{\infty}(f)(x)=\sup _{0<r<1} r \ln (1 / r)\left|(r f)_{r}(r x)\right| .
$$

Proposition 2.3. Let $f \in L_{p}\left(\Sigma_{n-1}\right)$ and $q=\max [p, 2]$. Then for $1<p \leqq \infty,\left\|g_{q}(f)\right\|_{p} \leqq A_{p, n}\|f\|_{p}$.

Proof. Case I. $1<p \leqq 2$. Then $q=2$ and

$$
g_{2}(f)(x) \leqq\left[\int_{0}^{1} r^{2} \ln (1 / r)\left|f_{r}(r x)\right|^{2} d r\right]^{1 / 2}+\left[\int_{0}^{1} r \ln (1 / r)|f(r x)|^{2} d r\right]^{1 / 2}
$$

The second integral is bounded by $B_{p, n} f^{*}(x)$. Since $\left|f_{r}(r x)\right| \leqq$ $|\nabla f(r x)|$, the first integral is bounded by $g(f)(x)$.

Thus $g_{2}(f)(x) \leqq B_{p, n} f^{*}(x)+g(f)(x)$ and so by Proposition 2.1, $g_{2}(f)(x) \in L_{p}\left(\Sigma_{n-1}\right)$ and $\left\|g_{2}(f)(x)\right\|_{p} \leqq B_{p, n}\left\|f^{*}\right\|_{p}+\|g(f)(x)\|_{p} \leqq A_{p, n}\|f\|_{p}$. 


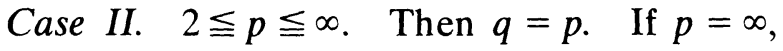

$$
\|g(f)(x)\|_{\infty}=\left\|\sup _{0<r<1} r \ln (1 / r)\left|(r f)_{r}(r x)\right|\right\|_{\infty, d x} \leqq A_{p, n}\|f\|_{\infty} .
$$

If $p<\infty$, then

$$
\begin{aligned}
g_{p}(f)(x) & =\left[\int_{0}^{1}\left|r \ln (1 / r)(r f)_{r}(r x)\right|^{2}\left|r \ln (1 / r)(r f)_{r}(r x)\right|^{p-2} d r / r \ln (1 / r)\right]^{1 / 2} \\
& \leqq C_{p, n}\left[f^{*}(x)\right]^{(p-2) / 2}\left[\int_{0}^{1}\left|r \ln (1 / r)(r f)_{r}(r x)\right|^{2} d r / r \ln (1 / r)\right]^{1 / p} \\
& =C_{p, n}\left[f^{*}(x)\right]^{(p-2) / 2} g_{2}(f)(x)^{2 / p} .
\end{aligned}
$$

Hence $\left\|g_{p}(f)\right\|_{p}^{p} \leqq C_{p, n}\left\|f^{*}\right\|_{p}^{(p-2) / 2}\left\|g_{2}(f)\right\|_{p}^{2 / p} \leqq A_{p, n}\|f\|_{p}$ by Hölder's inequality, Proposition 2.2, and the argument above in Case I. We have the following converse.

Proposition 2.4. Let $f(r x)$ be harmonic on $B_{n}$. Let $q=\min [p, 2]$ and $g_{q}(f) \in L_{p}\left(\Sigma_{n-1}\right) . \quad f(r x)$ is the Poisson integral of a function $f(x) \in$ $L_{p}\left(\Sigma_{n-1}\right)$ and $\|f\|_{p} \leqq A_{p, n}\left\|g_{q}(f)\right\|_{p}$ for $1 \leqq p<\infty$.

We will need the following lemmas.

LEMma 2.1. Let $f \in L_{p}\left(\Sigma_{n-1}\right)$ and $h \in L_{p}\left(\Sigma_{n-1}\right)$ where $1 / p+1 / p^{\prime}=1$ and $1<p<\infty$. Then

$$
\int_{\Sigma_{n-1}} f(x) g(x) d x=4 \int_{\Sigma_{n-1}} \int_{0}^{1} r \ln (1 / r)(r f)_{r}(r x)(r h)_{r}(r x) d r d x .
$$

Proof. It suffices to prove this for $f \in L_{p}\left(\Sigma_{n-1}\right)$ and $g \in C^{\infty}\left(\Sigma_{n-1}\right)$ since $C^{\infty}\left(\Sigma_{n-1}\right)$ is dense in $L_{p},\left(\Sigma_{n-1}\right)$. Let $0<s<1$. Then

$$
\int_{\Sigma_{n-1}} f(x) h(\grave{x}) d x=\lim _{s \rightarrow 1^{-}} \int_{\Sigma_{n-1}} f(s x) h(s x) d x .
$$

Let $f(s x)=\sum_{k, l} a_{k l} S^{k} Y_{l}^{(k)}(x)$ and $h(s x)=\sum_{k, l} b_{k l} S^{k} Y_{l}^{(k)}(x)$ where $b_{k l}=$ $O\left(k^{-t}\right)$ for all $t$ since $h \in C^{\infty}\left(\Sigma_{n-1}\right)$.

Thus $\int_{\Sigma_{n-1}} f(x) g(x) d x=\Sigma_{k, l} a_{k l} b_{k l}$.

An easy argument shows that

$$
4 \int_{\Sigma_{n-1}} \int_{0}^{1} r \ln (1 / r)(r f)_{r}(r x)(r h)_{r}(r x) d r d x=\sum_{k, l} a_{k l} b_{k l} .
$$


REMARK. This Lemma shows that the map $f \rightarrow \frac{1}{2} g_{2}(f)$ is an isometry of $L_{2}\left(\Sigma_{n-1}\right)$.

Lemma 2.2. Let $f \in L_{p}\left(\Sigma_{n-1}\right)$ and $f(r x)$ be its Poisson integral. Let $q=\min [p, 2] \quad$ and $g_{q}(f) \in L_{p}\left(\Sigma_{n-1}\right)$. Then $\|f\|_{p} \leqq A_{p, n}\left\|g_{p}(f)\right\|_{p}$ for $1 \leqq p<\infty$.

Proof. Case I. $p=1 . \quad r f(r x)=\int_{0}^{r}(\rho f)_{\rho}(\rho x) d \rho$.

So $\quad|r f(r x)| \leqq \int_{0}^{1}\left|(\rho f)_{\rho}(\rho x)\right| d \rho \leqq g_{1}(f)(x)$. Thus $\quad\|r f(r x)\|_{1, d x} \leqq$ $\left\|g_{1}(f)\right\|_{1}$ and so $\|f\|_{1} \leqq\left\|g_{1}(f)\right\|_{1}$.

Case II. $1<p \leqq \infty$. Then $q=p . \quad\|f\|_{p}=\sup \int_{\Sigma_{n-1}} f(x) h(x) d x$ where the sup is over all $h \in L_{p^{\prime}}\left(\Sigma_{n-1}\right)$ where $\|h\|_{p^{\prime}}=1$ and $1 / p+1 / p^{\prime}=1$.

By Lemma 2.1,

$$
\begin{aligned}
& \left|\int_{\Sigma_{n-1}} f(x) h(x) d x\right|=\left|4 \int_{\Sigma_{n-1}} \int_{0}^{1} r \ln (1 / r)(r f)_{r}(r x)(r h)_{r}(r x) d r d x\right| \\
& \quad \leqq 4 \int_{\Sigma_{n-1}} g_{p}(f)(x) g_{p^{\prime}}(f)(x) d x \leqq 4\left\|g_{p}(f)\right\|_{p}\left\|g_{p^{\prime}}(h)\right\|_{p^{\prime}} \\
& \quad \leqq A_{p, n}\left\|g_{p}(f)\right\|_{p}\|h\|_{p^{\prime}}=A_{n, p}\left\|g_{p}(f)\right\|_{p}
\end{aligned}
$$

by Proposition 2.3 since $p^{\prime} \geqq 2$.

Case III. $2 \leqq p<\infty$. Then $q=2$. An argument similar to the one above applies here.

\section{Proof of Proposition 2.4.}

Case I. $p=1$. In view of Lemma 2.2, it suffices to show that $f(r x)$ is the Poisson Integral for a function in $L_{1}\left(\Sigma_{n-1}\right) \cdot r f(r x)=\int(\rho f)_{\rho}(\rho x) d \rho$ and so $\|r f(r x)\|_{1, d x} \leqq\left\|g_{1}(f)(x)\right\|_{1}$. The result now follows from an easy argument.

Case II. $1<p<\infty$. In view of Lemma 2.2, it suffices to show that $f(r x)$ is the Poisson Integral of a function in $L_{p}\left(\Sigma_{n-1}\right)$. An easy variant of Lemma 2.2 shows that if $f \in L_{p}\left(\Sigma_{n-1}\right)$, then $\|f\|_{p} \leqq A_{p, n}\left\|g_{2}(f)\right\|_{p}$ for $1<p<\infty$. A straightforward argument finishes this part of the proof.

We have the following easy corollaries. 
COROLlaRY 2.1. Let $f(r x)$ be harmonic for $x \in \Sigma_{n-1}, 0 \leqq r<1$, and let $g_{2}(f) \in L_{p}\left(\Sigma_{n-1}\right)$ for $1<p<\infty$. Then $f(r x)$ is the Poisson integral of a function $f(x) \in L_{p}\left(\Sigma_{n-1}\right)$ and $\|f(x)\|_{p} \leqq A_{p, n}\left\|g_{2}(f)(x)\right\|_{p}$.

COROllary 2.2. Let $f(r x)$ be harmonic for $x \in \Sigma_{n-1}, 0 \leqq r<1$, and let $g(f) \in L_{p}\left(\Sigma_{n-1}\right)$ for $1<p<\infty$. Then $f(r x)$ is the Poisson integral of a function $f(x) \in L_{p}\left(\Sigma_{n-1}\right)$ and $\|f(x)\|_{p} \leqq A_{p, n}\|g(f)(x)\|_{p}$.

Proposition 2.5. (a) $L_{p, \alpha} \subset \Lambda(\alpha ; p, q), q=\max [p, 2], 1<p \leqq \infty$.

(b) $\Lambda(\alpha ; p, q) \subset L_{p, \alpha}, q=\min [p, 2], 1 \leqq p<\infty$.

(c) $L_{1, \alpha} \subset \Lambda(\alpha ; 1, \infty)$

(d) $\Lambda(\alpha ; \infty, 1) \subset L_{x, \alpha}$.

The inclusion maps are continuous.

Proof. With minor changes the proof of Theorem 15 of Taibleson $[7 ;$ p. 452] can be apllied here to give the result.

\section{REFERENCES}

1. H. Greenwald, Lipschitz spaces on the surface of the unit sphere in Euclidean n-space, Pacific J. Math., 50 (1974), 63-80.

2. D. L. Ragozin, Polynomial approximation and harmonic analysis on spheres, Seminar Report, Mass. Inst. of Tech., 1968.

3. R. T. Seeley, Spherical harmonics, No. 11 of H. Ellsworth Slaught Memorial Papers, Amer. Math. Monthly, 73, No. 4 (1966), 115-121.

4. E. M. Stein, On the functions of Littlewood-Paley, Lusin, and Marcinkiewicz, Trans. Amer. Math. Soc., 88 (1958), 430-466.

5. - Topics in harmonic analysis related to the Littlewood-Paley theory, Annal of Mathematica Studies, No. 63, Princeton University Press, 1970.

6. E. M. Stein and G. L. Weiss, Introduction to Fourier Analysis on Euclidean Spaces, Princeton University Press, 1971.

7. M. H. Taibleson, On the theory of Lipschitz spaces of distributions on Euclidean $n$-space, I, principal properties, J. Math. and Mech., 13, No. 13, (May 1964), 407-480.

8. A. Zygmund, Trigonometric Series, Sec. Ed., Cambridge, 1968.

Received September 2, 1976.

California Polytechnic State University - San luis Obispo 



\section{Pacific Journal of Mathematics \\ Vol. 70, No. $1 \quad$ September, 1977}

William H. Barker, Noether's theorem for plane domains with hyperelliptic

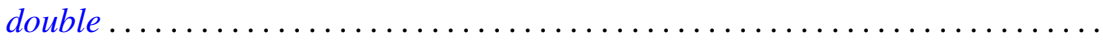

Michael James Beeson, Non-continuous dependence of surfaces of least area on the boundary curve ...................................... 11

Horst Behncke, Functions acting in weighted Orlicz algebras . . . . . . . . . . . . 19

Howard Edwin Bell, A commutativity study for periodic rings . . . . . . . . . . . 29

Peter Botta and Stephen J. Pierce, The preservers of any orthogonal group ....... 37

Douglas S. Bridges, The constructive Radon-Nikodým theorem ............. 51

James Dennis Brom, The theory of almost periodic functions in constructive

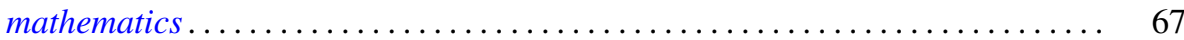

N. Burgoyne and C. Williamson, Semi-simple classes in Chevalley type groups ....

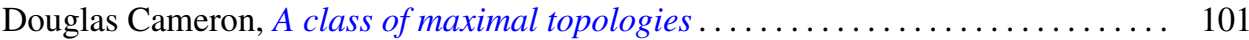

L. Carlitz, Enumeration of doubly up-down permutations . . . . . . . . . . . . . . 105

Paul Robert Chernoff, The quantum n-body problem and a theorem of

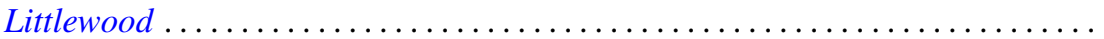

Jo-Ann Deborah Cohen, Locally bounded topologies on $F(X) \ldots \ldots \ldots \ldots \ldots \ldots$

Heinz Otto Cordes and Robert Colman McOwen, Remarks on singular elliptic

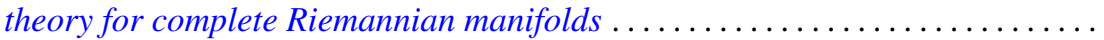

Micheal Neal Dyer, Correction to: "Rational homology and Whitehead

products"

Robert Fernholz, Factorization of Radonifying transformations

Lawrence Arthur Fialkow, A note on quasisimilarity. II ...... . .

Harvey Charles Greenwald, Lipschitz spaces of distributions on the surface of unit

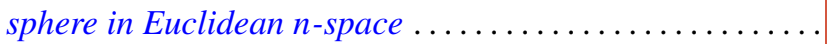

Albrecht Irle, On the measurability of conditional expectations

Tom (Roy Thomas Jr.) Jacob, Matrix transformations involving simple sequence

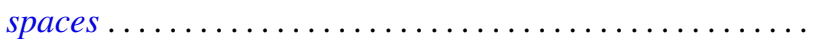

A. Katsaras, Continuous linear maps positive on increasing continuous

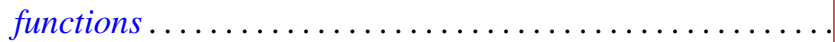

Kenneth Kunen and Judith Roitman, Attaining the spread at cardinals of cofinality

Lawrence Louis Larmore and Robert David Rigdon, Enumerating normal bundles

of immersions and embeddings of projective spaces ...... . .

Ch. G. Philos and V. A. Staïkos, Asymptotic properties of nonoscillatory solutions of differential equations with deviating argument .

Peter Michael Rosenthal and Ahmed Ramzy Sourour, On operator algebras containing cyclic Boolean algebras...

Polychronis Strantzalos, Strikt fast gleichgradig-stetige und eigentliche

Aktionen ...

Glenn Francis Webb, Exponential representation of solutions to an abstract

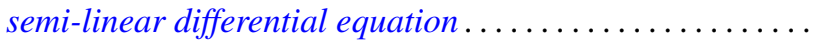

